\title{
Characterizing Intervention Strategies Used in Community-Based Mental Health Care for Infants and their Families
}

\section{Authors:}

Gabriela M. Rodríguez, PhD, Visiting Assistant Professor, Indiana University School of Medicine

Dainelys Garcia, PhD, Assistant Professor, University of Miami, Miller School of Medicine Angela Blizzard, MS, Graduate Student, Florida International University

Nicole E. Barroso, MS, Graduate Student, Florida International University

Daniel M. Bagner, PhD, Associate Professor, Florida International University

Date of submission: 03/21/2017

Contact author: Gabriela M. Rodríguez, PhD, 705 Riley Hospital Drive, Suite 4300, Indianapolis, IN 46202. Phone: 317-944-8162. Email: gabrodri@iu.edu

The current research has not been presented at a meeting. This research is based on a dissertation by Gabriela M. Rodríguez completed at Florida International University, supervised by Daniel M. Bagner.

\section{Compliance with Ethical Standards:}

This study was not funded. All authors declare that they have no conflict of interest.

Ethical approval: All procedures performed in studies involving human participants were in accordance with the ethical standards of the institutional and/or national research committee and with the 1964 Helsinki declaration and its later amendments or comparable ethical standards. Informed consent: Informed consent was obtained from all individual participants included in the study.

This is the author's manuscript of the article published in final edited form as: Rodríguez, G. M., Garcia, D., Blizzard, A., Barroso, N. E., \& Bagner, D. M. (2018). Characterizing Intervention Strategies Used in Community-Based Mental Health Care for Infants and Their Families. Administration and Policy in Mental Health and Mental Health Services Research, 45(5), 716-730. https://doi.org/10.1007/s10488-018-0855-0 


\begin{abstract}
Mental health interventions for infants typically target high-risk groups and can prevent longterm negative outcomes. Despite federal initiatives promoting early intervention, minimal research has examined usual care services for infants, which is important to improve routine care. The current study characterized usual care practices in infant mental health through the adaptation and administration of a provider survey. Providers $(n=126)$ reported using a wide range of intervention strategies and few intervention programs with varied evidence. Findings can inform future research to identify quality improvement targets of usual mental health care for high-risk infants and their families.
\end{abstract}

Keywords: infant mental health; usual care; practice elements; early intervention 


\section{Characterizing Intervention Strategies Used in Community-Based Mental Health Care for Infants and their Families}

Infancy, defined herein as ages 0 to 3 years, is characterized by rapid growth within multiple environmental contexts, in which a variety of risk factors can lead to long-term negative outcomes (Pitzer, Esser, Schmidt, \& Laucht, 2010; Sameroff, 1998). Infancy presents an ideal opportunity for interventions to maximize young children’s potential for healthy social and emotional development (Blackman, 2002). Early intervention programs have targeted infants from high-risk groups and demonstrated positive outcomes (Olds, Sadler, \& Kitzman, 2007). However, minimal research has examined the nature of interventions implemented in community-based services for infants. Information about usual care, including the range of intervention approaches used, is essential to maximize the impact of early intervention and identify targets for improvement. Thus, the purpose of the current research study was to characterize usual mental health care for infants, which is an important first step towards enhancing community-based mental health care for infants and their families.

\section{Risk Factors in Infancy Predict Long-Term Negative Outcomes}

Early multiple risk factors place infants at risk for subsequent mental health problems. For example, early disturbances in the parent-infant relationship are associated with lower child involvement in the parent-child relationship at age 7 years (Easterbrooks, Biesecker, \& LyonsRuth, 2000) and externalizing behavior problems in middle childhood (Fearon, BakermansKranenburg, van IJzendoorn, Lapsley, \& Roisman, 2010). Furthermore, attachment problems in the parent-infant relationship are stable through early adulthood (Waters, Hamilton, \& Weinfield, 2000) and are associated with adult psychopathology (Sroufe, Carlson, Levy, \& Egeland, 1999). Poor parenting practices during infancy, including low parental warmth and involvement, low 
parental monitoring, and harsh and inconsistent discipline, are associated with subsequent child disruptive behavior (Burke, Loeber, \& Birmaher, 2002). Infant difficult temperament and attention problems, as well as oppositional, aggressive, and destructive behavior, during the first three years of life are associated with higher severity of conduct problems during the school-age years (Shaw, Owens, Giovannelli, \& Winslow, 2001).

In addition to their individual effects, early risk factors often co-occur and are interrelated. For example, when elevated maternal depressive symptomatology and high child fearlessness at age 2 years co-occur, they are associated with a trajectory of early-starter high conduct problems (Shaw, Gilliom, Ingoldsby, \& Nagin, 2003). Low socioeconomic status (SES) and parental substance abuse during infancy also have been shown to predict the onset of conduct disorder in adolescence (Loeber, Green, Keenan, \& Lahey, 1995). Furthermore, the effects of individual risk factors are small in comparison to the long-term negative effects of the accumulation of multiple risk factors. High-risk infants, such as infants of teenage mothers (Dubow \& Luster, 1990), infants born preterm (Aarnoudse-Moens, Weisglas-Kuperus, van Goudoever, \& Oosterlaan, 2009), infants with developmental delay (Baker, Blacher, Crnic, \& Edelbrock, 2002), and infants from low SES families (Sameroff, 1998), experience an accumulation of risk factors and are at significantly higher risk for mental health problems compared to infants with fewer risk factors (Sameroff, 1998).

\section{Policymakers Fund Early Intervention despite High Variability in Evidence}

Research demonstrating the long-term effects of risk factors during infancy has contributed to policymakers prioritizing early intervention. For example, the Patient Protection and Affordable Care Act of 2010 authorized the Maternal, Infant, and Early Childhood Home Visiting Program, a policy initiative facilitating collaboration at multiple levels (i.e., federal, 
state, and local) to improve health and developmental outcomes for at-risk infants through homevisiting programs. The program requires that grantees demonstrate improvement in various benchmark areas, including improved maternal and newborn health; prevention of child injuries or maltreatment; and improvement in child school readiness and achievement. In addition, the authorizing legislation requires that at least $75 \%$ of grant funds are spent on one of the thirteen home visiting models meeting the evidence criteria set by the Department of Health and Human Services using the Home Visiting Evidence of Effectiveness (HomVEE) review of home visiting models (Avellar et al., 2016). However, the quality of the evidence supporting these programs varies, as studies of programs were not required to have undergone independent replication or to include fidelity standards for local implementing agencies. While these funding efforts have increased the availability of early intervention programs, the quality of interventions for infants implemented in usual care settings has yet to be rigorously examined.

\section{Usual Care Research in Childhood and Infancy}

Despite federal initiatives promoting early intervention, minimal research has examined community-based mental health services in infancy. In order to maximize community-based care during the critical period of infancy, we must first learn more about current practices (Kolko, 2006). Research on usual care (i.e., routine practice in community-based settings) provides reliable data on the range of treatment approaches, factors related to positive outcomes, and variations among locations, providers, and patients (Garland, Bickman, \& Chorpita, 2010). Although research on usual care in youth mental health has increased in recent years, studies have primarily included children older than 4 years.

In their 2010 article, McLeod and Weisz describe the development of the Therapy Process Observational Coding System for Child Psychotherapy-Strategies scale (TPOCS-S), an 
observational measure of strategies used in child and adolescent psychotherapy. Coders rated entire therapy sessions to measure the degree to which specific interventions were used by the therapists. Psychometric properties for the TPOCS-S were evaluated in a sample of 43 children (ages 8 to 15 years) receiving treatment for internalizing disorders in community mental health clinics, and the TPOCS-S was found to be reliable and valid. When comparing TPOCS-S subscales, McLeod and Weisz (2010) found that therapists used a variety of strategies and that these reflected a variety of theoretical orientations.

In one study of usual youth mental health care, Garland and colleagues (2010) obtained descriptive data and used an adapted version of the TPOCS-S to code therapists' use of intervention strategies during psychotherapy sessions for 191 children aged 4 to 13 years presenting with disruptive behavior disorders in six community clinics. Therapists were primarily marriage and family counselors (58\%), followed by psychologists (24\%), and social workers (18\%). Results indicated that while most children received a large number of sessions ( $M=22.4$ sessions), there was considerable variability in the specific treatment strategies used. Additionally, strategies were typically delivered at low intensity, indicating a likely incomplete application of strategies with limited follow-through. Finally, some strategies consistent with empirically-supported treatments were observed frequently (e.g., problem-solving skills, use of positive reinforcement) but others were rare (e.g., assigning or reviewing homework, role-play, or modeling), highlighting the discrepancy between empirically-supported treatments and usual care in youth mental health.

Brookman-Frazee and colleagues (2010) examined child, family, and therapist factors associated with therapist use of practice elements common to evidence-based practices by coding over 1,000 usual care psychotherapy sessions for children aged 4 to 13 years using an adapted 
version of the TPOCS-S. Results indicated that a significant proportion of the observed variability in therapist delivery of EBP was due to child, family, and therapist characteristics. Specifically, older children, children of caregivers with more education, children of caregivers with more alcohol use problems, and children whose therapists self-identified cognitivebehavioral or behavioral orientations received a higher proportion of EBP strategies. Additionally, results indicated relatively low use of EBP strategies overall.

Hurlburt and colleagues (2010) examined concordance between therapist self-rated use of strategies and observer ratings in a sample of 4-13 year-old children receiving outpatient community-based services for behavior problems. After each session, therapists completed the Child Therapy Process Rating System (CTPRS) checklist to provide information regarding the goals and strategies pursued in the session. Observers later coded the same information while watching videotaped sessions using the CTPRS observational coding system. Results indicated poor concordance between therapist self-ratings and observer ratings, as therapists generally reported higher frequency and intensity of goals and strategies used per session compared to observational coders. These studies highlight the need for future multimethod research, as well as the need for research examining usual care in younger children.

To our knowledge, only one study to date has examined usual mental health care practices with infants. Macdonald and colleagues (2005) conducted semi-structured interviews with staff from 18 programs focused on children under 2 years in South Brisbane, Australia. Services addressed a variety of concerns, such as neonatal health, infant development, infant protection and safety, maternal health, and parent support. Infants were targeted for intervention on the basis of at-risk status because of poor health, developmental disability, infant abuse and neglect, family violence, maternal substance abuse, maternal mental health problems, poverty, or 
cultural/linguistic background associated with elevated risk for adverse outcomes. Only four of the 18 programs focused on the needs of both parents and infants. Providers reported increased emphasis on parenting knowledge and skills and infant physical development and safety relative to the provision of infant mental health services. Results indicated that services were fragmented, lacked continuity and communication between other services, and rarely included the parents and infant together. However, current practices in community-based usual care for infants in the United States remain unexamined.

\section{Current Study}

The current study aims to address the knowledge gap that exists as a result of the dearth of research on usual care practice in children's mental health (Bickman, 2000; Hoagwood \& Kolko, 2009), which is especially striking for infants. Practice-based research yielding descriptive information about the range of usual practices outside of research contexts is essential to bridging the research-to-practice gap. To our knowledge, the current study is the first examination of community-based usual mental health care services for infants in the United States. Through a two-phase survey of community mental health clinicians, the primary purpose of this research was to characterize usual mental health care for infants and toddlers via descriptive data on the intervention strategies and programs most commonly utilized. For the purpose of the current study, mental health services for infants and their families were defined as “services focusing on social, emotional, and/or behavioral health.

During Phase I, a small number of community mental health clinicians $(n=5)$ contributed to the adaptation of a measure of intervention strategies and the development of an online survey describing current practices in infant mental health. During Phase II, a separate and large sample of community mental health clinicians $(n=126)$ completed the online survey and 
provided information about the range of practices they use. Descriptive information about the intervention strategies and programs used by providers of usual mental health care for infants and their families was provided as a first step toward maximizing the quality of existing services.

\section{Method}

\section{Phase I}

Participants. Five mental health providers within an Infant Mental Health program at a community mental health agency participated in Phase I of the study. Participants were all female $(100.0 \%)$ with a mean age of 41.4 years $(S D=10.3$ years, Range $=27$ to 51 years). Three participants were master's-level clinicians (60.0\%), and two were doctoral-level clinicians (40.0\%). All participants were providing mental health services to at least one child aged 0 to 3 years and his or her family at the time of the discussion group and reported to have provided services to infants and their families for an average of 10.8 years $(S D=7.4$ years, Range $=6$ to 24 years).

Measure adaptation. Prior to the start of Phase I, the authors and three experts in infant mental health research, early childhood mental health research, and child mental health services research conducted an iterative review of the Hawaii Child and Adolescent Mental Health Division (CAMHD) Service Provider Monthly Treatment and Progress Summary (Hawaii, 2008), an existing measure of 63 treatment strategies used in child and adolescent mental health. The purpose of this review was to remove any strategies considered to be irrelevant to mental health care for infants (e.g., twelve-step program) in order to maximize provider time during the subsequent facilitated discussion groups described below. Intervention strategies, organized in the original measure in alphabetical order, were organized into strategies that are typically used directly with the parent (e.g., psychoeducation), with the parent and infant together (e.g., play 
therapy, ignoring/differential reinforcement), or directly with the infant/general strategies (e.g., therapist praise/rewards, relationship/rapport building).

The CAMHD measure was selected as a starting point for the current study for several reasons. First, intervention strategies are considered the unit of interest. The intermediate level of analysis is ideal for practice-based research because it is more specific than examining theoretical orientations but broader and more practical than classifying individual therapist utterances (Garland, Hurlburt, Brookman-Frazee, Taylor, \& Accurso, 2010). Second, the CAMHD measure was designed for children and adolescents, so it provided the best starting point for adaptation to infants. Third, it contains clear operationalized definitions of each intervention strategy, includes a large variety of intervention strategies, and has been used as a starting point in previous research to examine usual care practices with adolescents (BearsleySmith, Sellick, Chesters, \& Francis, 2008). As developing a measure was not the primary aim of the current study, the existing measure was adapted through the aforementioned iterative expert review process followed by two facilitated discussion groups with a small number of providers from a local community mental health agency.

Facilitated discussion group 1. Five providers attended the first facilitated discussion group. The primary aim was to bring providers together to focus on intervention strategies they used on a regular basis with their clients aged 0 to 3 years and their families. Providers reviewed the adapted version of the CAMHD Service Provider Monthly Treatment and Progress Summary (Hawaii, 2008) and participated in a facilitated discussion, led by the first author, regarding their use of these intervention strategies in their current treatment of infants. Providers were encouraged to comment on the applicability of the intervention strategies, as defined, to their practice and to suggest further revisions to enhance the comprehensiveness of the measure for 
infants or indicate whether no further revisions were needed. Following procedures used by Nicolaidis and colleagues (2011), provisional changes to the measure in terms of relevance to infant mental health practice were made during the discussion group using the consensus built among providers using the five-finger decision method. All providers verbally rated their agreement with each proposed change to the definition of each intervention strategy on a 5-point scale (from 1 = approve to 5 = disapprove). If any providers rated the proposed change a 3 or higher, indicating they did not approve, participants were asked to continue the discussion and modify the changes. Participants then were asked to rate their agreement with the modified changes. This process continued until all participants rated the proposed change as a 1 or 2 on the scale, indicating agreement. In order to enhance the fluidity of the discussion, the facilitator did not collect data on provider ratings during the discussion.

Participants were also presented a list of names of 36 early childhood intervention programs that were obtained from the Substance Abuse and Mental Health Services Administration (SAMHSA) National Registry of Evidence-based Programs and Practices (NREPP), a searchable online database of mental health and substance abuse interventions. Interventions included in this registry underwent independent assessment by certified NREPP reviewers and were rated according to NREPP guidelines. All interventions listed as applicable in early childhood, defined by NREPP as ages 0 to 5 years, were included. Participants were encouraged to suggest additional early childhood intervention programs. Ten additional programs that were not included in the original list of 36 programs (e.g., Greenspan’s Floor Time approach) were identified by participants and added to the list. The evidence base for the ten added programs varies. For example, one of the ten programs added by participants (i.e., Conscious Discipline) was subsequently reviewed and added to NREPP, while another added 
program (i.e., Prolonged Parent Child Embrace (PPCE) Therapy or “Holding Therapy”) has been identified as a potentially harmful treatment (Mercer, 2013).

Survey development. The revisions recommended by providers were used to further adapt the Hawaii CAMHD measure to capture intervention strategies used in usual mental health care for infants. The tailored design method (Dillman, Smyth, \& Christian, 2014) was used to develop a survey to examine practice elements (using the adapted CAMHD measure) and modes of intervention delivery, to be used in the second phase of the study. The tailored design method encourages participation through building trust with the research team and increasing the benefits and decreasing the costs of participation (Dillman et al., 2014). The survey was prepared for administration using the online Qualtrics electronic survey platform. The cover letter e-mail sent to potential participants contained an anonymous survey link. Inclusion criteria included participants affirming they provide mental health services and agreeing to participate in the study. As the survey was only provided in English, potential participants were also required to read English to complete the survey.

Facilitated discussion group 2. A second facilitated discussion group was conducted in order to pilot the survey and finalize content. Three of the five providers who participated in the first discussion group also participated in the second discussion group. Procedures recommended by Bowden and colleagues (2002) were used to assess the validity of survey items through a discussion group. Specifically, participants were shown each item along with a description of the intended meaning for each item. Feedback was elicited regarding each item, such as whether each item conveyed the intended meaning and whether response options were clear. Feedback was utilized to make minor revisions and finalize the survey.

\section{Phase II}


Participants. One hundred twenty-six mental health professionals participated in the online survey. Participants were primarily female (96.0\%) with a mean age of 41.6 years $(S D=$ 11.9, Range $=24$ to 70 years). Most participants were master’s-level clinicians (65.9\%), followed by doctoral-level clinicians (26.2\%) and bachelor-level clinicians (7.9\%). Likely due to the recruitment methods described below, most participants were located in the state of Florida ( $n=88,69.8 \%)$. The remaining participants were located in the following states: Colorado $(n=$ 12, 9.5\%), Illinois ( $n=8,6.3 \%)$, Massachusetts $(n=4,3.2 \%)$, Maine ( $n=2,1.6 \%)$, New Mexico $(n=2,1.6 \%)$, Oregon $(n=2,1.6 \%)$, Arizona $(n=1,0.8 \%)$, Connecticut $(n=1,0.8 \%)$, Iowa ( $n=1,0.8 \%)$, Louisiana $(n=1,0.8 \%)$, New Hampshire $(n=1,0.8 \%)$, Virginia $(n=1$, 0.8\%), and Wyoming ( $n=1,0.8 \%)$. One participant did not identify her location.

Participants described their caseloads as consisting, on average, of 53.1\% (SD = 19.1) male clients, 63.3\% $(S D=33.7)$ clients from low-income families, 56.5\% $(S D=28.4)$ clients from ethnic/racial minorities, $42.9 \%(S D=35.6)$ clients aged 0 to 3 years, $35.9 \%(S D=29.1)$ clients aged 4 to 5 years, and 44.9\% (SD = 31.5) clients aged 6 years and older. Additionally, participants indicated their caseloads, on average, consisted of $85.9 \%(S D=23.7)$ Englishspeaking families and 22.9\% (SD = 27.9) Spanish-speaking only families.

Recruitment. To our knowledge, no state or national infant mental health clinician lists or databases exist, so the infant mental health clinician population is a hidden population and a random sample cannot be drawn. Therefore, participants for Phase II of the current study were recruited through several methods, including direct e-mail contact, study flyers distributed at an international conference, presentations at local mental health professional groups, infant mental health distribution lists, and use of chain-referral sampling. In order to ensure there were enough participants who provided services to infants, we focused our recruitment efforts toward early 
childhood groups, when possible. Direct email addresses were obtained through publicly available online listings (e.g., Psychology Today "Find a therapist” tool). Participants who stated in their online listing they provided services to children and had a listed e-mail address were contacted. An email cover letter with a link to the online survey was sent to 346 potential participants. Direct contact methods focused on potential participants locally within the state of Florida. With permission from conference hosts, flyers including a link to the online survey were distributed at the Miami International Child and Adolescent Mental Health (MICAMH) Conference, which is attended by many local front-line mental health providers of young children given its focus on evidence-based prevention and treatment for children with mental health problems. The first author also presented the proposed study and distributed survey flyers during regularly scheduled meetings to local mental health professional groups (e.g., the Young Children with Special Needs and Disabilities Council, the Miami chapter of the Florida Association for Infant Mental Health).

In addition to recruitment efforts in Florida, other state infant mental health associations were contacted via e-mail and asked to distribute a cover letter with a link to the online survey via e-mail to their distribution lists. Thirty associations were contacted, and 14 indicated they distributed the link. Finally, chain-referral sampling was used to expand the initial sample. Clinicians who participated in the online survey were asked if they would be willing to pass along information about the study they just completed to other potential participants. In order to protect privacy, participants were asked to forward information about the survey to colleagues who might be interested in participating in the study. Participants did not receive incentives or compensation for referrals. 
In the final survey, participants were asked to report how they learned about the study. Most participants indicated they learned about the study via direct e-mail contact $(n=45$, $35.7 \%)$, followed by a distribution list ( $n=40,31.7 \%)$, a colleague ( $n=31,24.6 \%)$, a supervisor ( $n=7,5.5 \%)$, or a conference or presentation flyer $(n=2,1.6 \%)$. One participant did not identify how she learned about the study. As a result of the recruitment efforts used, it is unknown how many potential participants were contacted; thus, a response rate cannot be determined. Comparison of the number of participants who indicated they were recruited through email $(n=45)$ to the number of potential participants who were sent direct survey e-mails $(n=$ 346) yields a response rate of 13\%. Though this may be the best estimate of response rate in the current study, it does not account for inactive e-mail addresses or undeliverable e-mails. A previous online survey of mental health care providers estimated a higher response rate (21.9\%; Nelson \& Steele, 2007). However, both in this previous study and the current study, an actual response rate could not be determined, as the number of potentially eligible participants was unknown.

Measures. Participants completed the adapted Hawaii CAMHD measure, which contained a list of intervention strategies (e.g., activity scheduling, care coordination), and selected which strategies they use with the parent in reference to the infant, the parent and infant together, and directly with the infant. A brief description for each strategy (obtained from the original measure) was provided to participants. For strategies that participants indicated they used with infants, participants were also asked to rate the percentage of families with which they use each strategy and the amount of time (within a typical 1-hour session) they typically spend on each strategy. Participants were asked to select factors which influence their choice of 
intervention strategy and to rank order the selected factors in terms of amount of influence.

Participants also rated how often they use each listed intervention program with infants.

\section{Intervention Strategies}

\section{Results}

Strategies used with parent in reference to infant. As illustrated in Table 1, participants most frequently endorsed parent coping (96.1\%), parent psychoeducation (91.3\%), communication skills (89.4\%), and ignoring/differential reinforcement of other behavior (83.5\%) as strategies they used with parents in reference to their infants. Among the strategies endorsed, parent psychoeducation $(M=89.8 \%, S D=17.7)$, skill building $(M=83.5 \%, S D=22.2)$, problem solving $(M=83.0 \%, S D=22.6)$, and parent coping $(M=82.2 \%, S D=22.1)$ were reported to be used with the largest percentage of families of infants. Participants indicated they spent the most amount of time within a typical 60-minute session using skill building $(M=29.2$ minutes, $S D=16.0)$, parent psychoeducation $(M=27.8$ minutes, $S D=16.5)$, and problem solving $(M=26.3$ minutes, $S D=16.1)$.

Strategies used with parent and infant. The intervention strategies used with parents and their infant which participants most frequently endorsed were family engagement (87.0\%), attending (76.6\%), therapist praise/rewards (75.5\%), and play therapy (74.2\%). Among the strategies endorsed, family engagement $(M=79.3 \%, S D=26.5)$ and family therapy $(M=$ $70.3 \%, S D=31.9$ ) were used with the largest percentage of families. Participants indicated they spent the most amount of time within a typical 60-minute session using play therapy $(M=36.0$ minutes, $S D=16.5)$, family therapy $(M=33.2$ minutes, $S D=18.4)$, and family engagement $(M$ $=32.4$ minutes, $S D=17.0)$. 
Strategies used directly with infant or general strategies. The intervention strategies used directly with infants or general strategies which participants most frequently endorsed were relationship/rapport building (95.5\%), care coordination (79.8\%), and supportive listening (78.7\%). Among the strategies endorsed, supportive listening $(M=90.0 \%, S D=21.4)$ and relationship/rapport building $(M=89.3 \%, S D=22.9)$ were used with the largest percentage of families by providers. Participants indicated they spent the most amount of time within a typical 60 -minute session using supportive listening $(M=40.4$ minutes, $S D=16.7)$ and relationship/rapport building $(M=38.5$ minutes, $S D=17.2)$.

\section{Factors Influencing Choice of Intervention Strategy}

As shown in Table 2, participants endorsed the following factors as the most influential in their choice of intervention strategy: family culture (91.3\%), participants’ own knowledge of intervention strategy (88.0\%), caregiver/child cognitive ability (83.7\%), and results of assessment (80.4\%). Court-ordered use of intervention strategy (10.9\%) and use of intervention strategy by respected colleagues (38.0\%) were least frequently endorsed as influential in participants' choice of intervention strategy. In addition, eight participants (8.7\%) added a factor for the “other” response, including "level of family stress," “data collected," and "needs of the child and family.” Participants also rank ordered the factors they selected as influential in their choice of intervention strategy $(1=$ most influential to 5 = least influential). Overall, participants ranked results of assessment $(M=2.1, S D=1.3)$ and family culture $(M=2.4, S D=1.1)$ as the most influential factors. Use of intervention strategy by respected colleagues $(M=5.4, S D=1.3)$ and court-ordered use of strategy $(M=5.0, S D=2.3)$ were ranked as the least influential factors. For the eight participants who wrote in an additional factor, they ranked the written-in factor as most influential $(M=1.8, S D=1.0)$. 


\section{Early Childhood Intervention Programs}

Participants were presented the list of 46 intervention programs and asked to rate how often they use each program with infants on a 4-point scale (never, occasionally, very often, always). As illustrated in Table 3, more than 20\% of participants endorsed using six programs “very often” or “always” with infants: Active Parenting, Child-Parent Psychotherapy (CPP), Speaking for Baby, Trauma-Focused Cognitive Behavioral Therapy (TF-CBT), Parent-Child Interaction Therapy (PCIT), and Circle of Security. All participants endorsed "never” using eight programs with infants: Chicago Parent Program, Children in Between, Family Spirit, Lesson One, ParentCorps, Partners with Families and Children: Spokane, Two Families Now: Effective Parenting Through Separation and Divorce (TFN), and Zippy’s Friends.

\section{Discussion}

The present study included an examination of current practices in usual mental health care for infants. Research on usual care practices is necessary to bridging the research-to-practice gap in children's mental health care (Garland, et al., 2010). Improving the effectiveness and efficiency of routine care requires close examination of current practices (Kolko, 2006). The current study contributes to this literature by providing descriptive data on provider use of intervention strategies and intervention programs with infants and their families. To our knowledge, this is the first study to describe these characteristics in usual mental health care for children younger than four years-old in the U.S.

\section{Intervention strategies}

Of the intervention strategies endorsed by most participants, three were used directly with the parent in reference to the infant (i.e., parent coping, parent psychoeducation, and communication skills), one was used with the infant and parent together (i.e., family 
engagement), and one was a general strategy (i.e., relationship/rapport building). The intervention strategies included in in the current study were also used in a study which applied the distillation and matching model to 322 randomized clinical trials for child mental health treatments (Chorpita \& Daleiden, 2009). Importantly, only 37 of the 322 trials included children aged 0 to 3 years. The two most commonly endorsed intervention strategies in the current study (i.e., parent coping and relationship/rapport building) were among the least common practice elements in evidence-based treatment protocols across problem areas in Chorpita and Daleiden’s (2009) study. Parent psychoeducation and communication skills, however, were commonly used strategies across many of the problem areas. It is possible that the relatively small number of trials including infants (less than 12\%) included in Chorpita and Daleiden’s study accounts for the discrepancy between that study and the current findings. Additionally, parent coping and rapport building may be used more often by clinicians with families of infants given the limited number of strategies that can be used directly with an infant as compared to a child or adolescent.

Of the five intervention strategies endorsed by fewer than $25 \%$ of participants, three were used directly with the infant (i.e., discrete trial training, exposure, and medication/ pharmacotherapy), and two were used with the parent in reference to the infant (i.e., response cost and catharsis). Three of the treatment strategies least commonly endorsed by providers in the current study were commonly used in evidence-based treatment protocols according to Chorpita and Daleiden’s (2009) study. For example, response cost was sometimes used for oppositional/aggressive behavior and to a lesser extent for attention deficit/hyperactivity. Discrete trial training was commonly used to treat autism spectrum disorder (ASD), and exposure was commonly used to treat anxiety and traumatic stress. Catharsis was not included in 
analyses in Chorpita and Daleiden's (2009) study due to low reliability, and medication was not included, as the study focused on psychosocial treatments.

It is possible that the low number of participants endorsing use of medication/ pharmacotherapy in the current study may reflect the professional characteristics of the current sample. For example, only one participant in the current study was a physician. Of the six participants who endorsed using medication/pharmacotherapy with infants, one was the physician, one was a doctoral-level clinician, and four were masters-level clinicians. Alternatively, a national study examining prescription rates in children aged 2- to 5-years-old estimated a psychotropic prescription rate of 1.0\% for this age group between 2006 and 2009 (Chirdkiatgumchai et al., 2013). Therefore, the low endorsement of medication treatment in the current sample reflects national trends. Exposure was also endorsed by few providers in the current study despite its common use in evidence-based protocols.

It is possible that few providers endorsed the use of exposure with infants due to a perception that younger children would not understand the process or hesitation to expose younger children to distressing stimuli. Additionally, it is possible that few clinicians endorsed the use of exposure with infants because exposure was not an appropriate approach for the presenting problem identified by the clinician. As data about the problem types addressed by clinicians in the current study were not obtained, it is not possible to know if clinicians were treating anxiety and not using exposure or not using exposure because they were not treating anxiety. To our knowledge, no studies have specifically identified practice elements in evidencebased interventions for infants. Therefore, the extent to which strategies endorsed for use with this population in the current study can be compared to evidence-based practice elements is 
limited. Future research should identify practice elements in evidence-based interventions for infants to compare with rates found in the current study.

\section{Factors Influencing Choice of Intervention Strategy}

The factors endorsed by most clinicians as influential in their choice of intervention strategy were family culture, caregiver/child cognitive ability, and clinician knowledge of intervention strategy. Most providers (91.3\%) in the current study indicated that family culture plays a role in their selection of intervention strategy. This finding suggests that clinicians may perceive some strategies as more or less applicable to families based on their cultural background. It is possible that a relative dearth of research examining evidence-based interventions among infants from diverse cultural backgrounds could have influenced this finding such that clinicians are not certain if evidence-based interventions will be effective with clients from minority groups. Thus, future research should examine how clinicians incorporate client family culture into their intervention selection, which may inform future research examining the implementation of evidence-based treatments for infants from diverse cultural backgrounds. Evidence-based treatments have been shown to be probably efficacious or possibly efficacious treatments with ethnic minority youth aged 5 years and older (Huey \& Polo, 2008), and emerging research has extended these findings to Mexican American children as young as 3years-old (McCabe \& Yeh, 2009; McCabe, Yeh, Lau, \& Argote, 2012). Therefore, it is possible that evidence-based interventions for infants are effective with ethnic minority infants.

Nevertheless, research examining the efficacy of interventions for infants should include racially- and ethnically-representative samples of infants. If such research suggests that evidence-based interventions are effective for infants from racial and ethnic minority families, this client characteristic may not be useful in guiding providers' choice of intervention strategy. 
Alternatively, if research suggests a differential intervention response for infants from different racial or ethnic groups, this characteristic could be helpful in providers' strategy selection. Additionally, it is possible that providers interpreted the term in various ways, such as family routines, family constellation, or family openness to change, because family culture was not explicitly defined in the current study. Therefore, future research should more clearly define family culture in order to disentangle these effects.

Many providers (83.7\%) also rated caregiver/child cognitive ability as an important factor to consider when selecting intervention strategies. On the one hand, research has demonstrated that caregivers with lower cognitive functioning may experience difficulty with parenting skillacquisition (Bagner \& Graziano, 2013; Tymchuk \& Andron, 1992). On the other hand, a randomized controlled trial examining the efficacy of a home-visiting parenting skills intervention with parents with intellectual disability demonstrated improvements in parent health and safety behaviors (Llewellyn, McConnell, Honey, Mayes, \& Russo, 2003). Some adaptations were made to the intervention to improve accessibility for the parents (e.g., additional graphics, simplified language), suggesting skill-acquisition difficulties in caregivers with intellectual disability can be ameliorated. Child cognitive ability may also play a role in the effectiveness of intervention strategies, though previous research has demonstrated that parent training interventions without adaptation can improve child problem behaviors (e.g., Bagner \& Eyberg, 2007). Therefore, while some minor adaptations to interventions may be warranted, it is unclear whether caregiver/child cognitive ability should guide providers’ choice of intervention strategy.

In addition to client characteristics, most providers (88.0\%) in the current study indicated that their own knowledge of intervention strategies affected their decision to use these strategies. This finding is consistent with research demonstrating that insufficient provider knowledge of 
evidence-based practices was a barrier to implementation and associated with lower use of these practices (Sanders, Prinz, \& Shapiro, 2009). Knowledge, defined as the exposure of an individual to the existence of an innovation and an understanding of how it functions, is the first stage described in Rogers’ (2010) model of the innovation-decision process by which change is implemented. This finding highlights the importance of training and education efforts, such as those demonstrated to increase provider knowledge of evidence-based practices (Lim, Nakamura, Higa-McMillan, Shimabukuro, \& Slavin, 2012).

The factors selected as influential by fewest providers (and ranked as least influential by providers who selected them) were court-ordered use of strategy and use of strategy by respected colleagues. The finding that court-ordered use of strategy was among the least influential for providers in the current study was surprising given that maltreatment victimization rates are highest for infants (U.S. Department of Health and Human Services, 2015). Younger children are more likely to be victims of maltreatment, however, they are less likely to receive services compared to older children (Garland, Landsverk, Hough, \& Ellis-MacLeod, 1996). Thus, it may be that while infants are overrepresented in the child welfare system (Malik, Crowson, Lederman, \& Osofsky, 2002), they may not be represented in the community mental health system at similar rates. However, as information about clinicians’ specific service sectors was not obtained, it is also possible the current study did not represent clinicians working in child welfare.

Few providers (38.0\%) in the current study indicated that the use of an intervention strategy by a respected colleague was influential in their choice of strategy. This finding is inconsistent with previous research suggesting that peer usage and satisfaction facilitates adoption of innovations (Frambach \& Schillewaert, 2002) and social diffusion theory, which 
suggests that persuading key opinion leaders facilitates the dissemination of innovations within their social networks (Rogers, 2010). It is possible that variations in participants' social networks account for the current finding. Providers who are embedded in organizations with large social networks and with opportunities for peer supervision, may be more likely to be influenced by their colleagues’ use of intervention strategies compared to providers with smaller social networks (e.g., solo private practice). Future research examining the extent to which organizational structure and/or supervision practices impact how peer usage of interventions influences clinician adoption of EBPs would be critical to the design of future dissemination efforts. Overall, future research should continue to examine factors that may influence clinical decision-making with infants, as knowledge about these factors could be used to inform training efforts (Jensen-Doss \& Hawley, 2010).

\section{Early Childhood Intervention Programs}

Active Parenting, the program reported to be used by most providers in the current study (60.5\%), is a video-based education program which emphasizes encouragement, building selfesteem, active listening, effective communication, and problem solving (Fashimpar, 2001). Three studies of Active Parenting (only one of which was published in a peer-reviewed journal) were included in NREPP's 2008 review of Active Parenting. However, only one of the unpublished studies included children ages 0 to 5 years, and the quality of research rating for this study was 2.2 (on a 0.0 to 4.0 scale; NREPP, 2008). For the other three frequently used programs, average quality of research ratings ${ }^{1}$ were 3.7 for Child-Parent Psychotherapy (CPP), 3.8 for Trauma-Focused Cognitive Behavioral Therapy (TF-CBT), and 3.3 for Parent-Child Interaction Therapy (PCIT). The other two frequently used programs (i.e., Speaking for Baby

\footnotetext{
${ }^{1}$ Quality of research ratings included in the current discussion were based on child outcomes in studies including children aged 0 to 5 years and averaged, when more than one study was included.
} 
and Circle of Security) were included based on suggestion from participants in Phase I of the current study, but these intervention programs have not been evaluated by NREPP.

Evidence-based early intervention programs typically target infants with identified riskfactors, such as infants from low SES families or infants born prematurely (Olds et al., 2007). For example, the Nurse-Family Partnership (NFP) is a nurse home visiting program for lowincome, first-time mothers during pregnancy and through the first 2 years of the child's life. In three large, randomized controlled trials, the NFP has been demonstrated to improve child and parent outcomes, including fewer injuries during early childhood and child arrests and convictions during adolescence, as well as higher rates of maternal employment (Olds, 2006). One randomized-controlled trial examined the relative impact of the NFP when delivered by nurses compared to paraprofessionals. However, no significant effects were observed on child and parent outcomes when delivered by paraprofessionals, whereas effects for families visited by nurses were consistent with the previous trials (Olds et al., 2002).

Another empirically-supported early intervention program is the Family Check-Up (FCU), a brief intervention for high-risk families (e.g., low SES, maternal depression, and/or elevated child problem behavior) and designed to prevent conduct problems by promoting consistent parent management practices and increasing caregiver involvement (Shaw, Dishion, Supplee, Gardner, \& Arnds, 2006). Randomized-controlled trials examining the FCU with families of infants under 2 years-old have demonstrated increased mother involvement in child behavior (e.g., mother keeps child in visual range), reduced child conduct problems (Dishion et al., 2008; Shaw \& Dishion, 2006), and improved inhibitory control and language development at age 4 years (Lunkenheimer et al., 2008). The FCU also has been demonstrated to have long-term effects on teacher-reports of child conduct problems at age 9.5 years (Shaw, 2015). 
However, despite strong evidence for the efficacy of the NFP and FCU, only $1.2 \%$ of providers in the current study reported using these programs “very often” or “always.” These findings suggest that while some infant mental health clinicians report using interventions with strong evidence, many clinicians do not necessarily implement intervention programs with the strongest quality of evidence. Additionally, some barriers to implementing these programs in community settings may account for this finding. The limited effectiveness of the NFP when implemented by paraprofessionals may limit its generalizability to implementation in community mental health clinics, as trained nurses may not be readily available and can be costly in such settings. While the FCU can be implemented by master's-level clinicians, who may be more readily available in community mental health centers, it is offered in only six states as of August 2015, according to the U.S. Department of Health and Human Services (2015). It is possible that the training, implementation, labor, materials, and infrastructure costs associated with some evidence-based programs are cost-prohibitive for many community mental health clinics.

\section{Limitations}

The current findings should be interpreted within the context of study limitations. First, the reliance on clinician self-report to estimate use of intervention strategy is one potential limitation, as previous research has found limited concordance between therapist self-rated use of strategies and observer ratings (Borntrager, Chorpita, Orimoto, Love, \& Mueller, 2013; Hurlburt, Garland, Nguyen, \& Brookman-Frazee, 2010). As discussed by Garland and colleagues (2010), although direct assessment of psychotherapy practice (e.g., live observation, audio- or video- recording and coding) is potentially more objective compared to indirect assessment (e.g., therapist and/or client self-report, chart/record review), it is also more costly. Therapist selfreport has been the most common method to examine psychotherapy practice (Garland et al., 
2010), likely due to its practical nature. Nevertheless, future research should attempt to utilize multiple assessment methods (e.g., therapist report and live observation or video coding; Hurlburt et al., 2010) to examine infant mental health practices in order to continue to examine concordance among methods.

Second, we did not use a random sample of the population of infant mental health clinicians because no state or national databases of these clinicians exist. Instead, the current study relied on several recruitment methods and nonprobability sampling. Additionally, to our knowledge, there are no published reports describing the characteristics of clinicians who typically provide services to infants. Because the current sample was not random and sample characteristics cannot be compared to population characteristics, it is not possible to determine whether the current sample is representative of the population of infant mental health providers. Therefore, it is critical that the current findings be interpreted with caution. Future research should characterize provider and practice characteristics in infant mental health in a nationally representative sample of providers.

Third, information about the types of problems targeted by clinicians in their intervention with infants was not obtained. Clinicians may address a wide range of issues within their practice with infants, so it is possible that the focus of intervention affected the intervention strategies used. Thus, future research should examine relations between problem types targeted and intervention strategies used for this age group. Fourth, the audience of some of the intervention strategies was changed during the adaptation process. For example, problem-solving and skillbuilding are intervention strategies designed to be used directly with children and are used as such in the existing literature. During the process of categorizing strategies, both problemsolving and skill-building emerged as strategies that could not be used directly with infants due 
to their very young age. However, clinicians in the discussion groups stated they might use these strategies with caregivers in reference to their infant and they were categorized as such. As the audience for these strategies in the current study differs from the audience in the existing literature, it is not possible to directly compare this finding to the evidence base. This limitation highlights the need for additional research examining evidence-based intervention strategies specifically for infants and their caregivers.

Fifth, participants were not required to view the definition of each intervention strategy (though each definition was available by hovering the mouse over the intervention strategy and this option was clearly stated in the instructions). Therefore, it is possible that participants did not view some or all of the definitions and rated their use of the strategy based on their own opinion or a more vernacular definition. For some strategies (e.g., problem-solving, skillbuilding) it is possible not requiring participants to read the intended audience contributed to their high endorsement rates. Six, the Hawaii CAMHD measure, which was designed to assess therapist use of EBP strategies with children and adolescents, was adapted for use with infants, rather than creating a new measure to assess the full range of individual intervention strategies that may be used with the target age group. Though creating a new measure would increase the likelihood that a broader range of potential strategies were examined and that all intervention strategies included were applicable to the target population, adapting the existing CAMHD measure was a beneficial approach.

Using an existing measure allows for placing the current findings in the context of the literature. Additionally, adapting the measure by removing items that were not applicable and slightly modifying the language for some items does not require generation and content assessment of new items. Furthermore, to complement the adapted list of intervention strategies, 
participants were asked to rate their use of specific intervention programs obtained from NREPP. In order to bridge the gap between the full range of potential programs and the programs included in NREPP, programs (e.g., Speaking for Baby, Circle of Security) were added to the initial list based on feedback from clinicians during the facilitated discussion groups. Finally, the descriptive nature of the study is a limitation. However, as discussed previously, descriptive data about usual care practices are a necessary first step to improving the quality of usual mental health care practices.

\section{Future Directions}

Despite these limitations, the current study provided important information about the range of intervention strategies and programs that characterize usual mental health care for infants. Future research should use a systematic approach, such as the distillation and matching model used by Chorpita and Daleiden (2009), to identify practice elements in evidence-based intervention protocols for infants. After evidence-based practice elements for infants are identified, they should be compared to the intervention strategies used in usual care. These comparisons will lead to the identification of effective existing services as well as quality improvement targets (i.e., areas where usual care diverges from empirically-supported treatments). In turn, this information can be used to design and implement quality improvement efforts with a focus on fit and sustainability. Future research should also assess the impact of these quality improvement interventions by comparing practices to the baseline benchmarks identified in the proposed research. Ultimately, this iterative intervention process will result in improved community-based mental health care for a population that, in addition to being one of the most vulnerable, could benefit most from intervention efforts. 


\section{References}

Aarnoudse-Moens, C. S. H., Weisglas-Kuperus, N., van Goudoever, J. B., \& Oosterlaan, J. (2009). Meta-analysis of neurobehavioral outcomes in very preterm and/or very low birth weight children. Pediatrics, 124(2), 717-728. https://doi.org/10.1542/peds.2008-2816

Avellar, S., Paulsell, D., Sama-Miller, E., Del Grosso, P., Akers, L., \& Kleinman, R. (2016). Home Visiting Evidence of Effectiveness Review: Executive Summary. Washington DC: Office of Planning, Research and Evaluation, Administration for Children and Families, U.S. Department of Health and Human Services.

Bagner, D. M., \& Eyberg, S. M. (2007). Parent-child interaction therapy for disruptive behavior in children with mental retardation: a randomized controlled trial. Journal of Clinical Child and Adolescent Psychology: The Official Journal for the Society of Clinical Child and Adolescent Psychology, American Psychological Association, Division 53, 36(3), 418-429. https://doi.org/10.1080/15374410701448448

Bagner, D. M., \& Graziano, P. A. (2013). Barriers to Success in Parent Training for Young Children with Developmental Delay: The Role of Cumulative Risk. Behavior Modification, 37(3), 356-377. https://doi.org/10.1177/0145445512465307

Baker, B. L., Blacher, J., Crnic, K. a, \& Edelbrock, C. (2002). Behavior Problems and Parenting Stress in Families of Three-year-old Children with and without Developmental Delays. American Journal of Mental Retardation : AJMR, 107(6), 433-444. https://doi.org/10.1352/0895-8017(2002)107<0433:BPAPSI>2.0.CO;2

Bearsley-Smith, C., Sellick, K., Chesters, J., \& Francis, K. (2008). Treatment content in child and adolescent mental health services: Development of the treatment recording sheet. 
Administration and Policy in Mental Health and Mental Health Services Research, 35(5), 423-435. https://doi.org/10.1007/s10488-008-0184-9

Bickman, L. (2000). The most dangerous and difficult question in mental health services research. Mental Health Services Research, 2(2), 71-72.

Blackman, J. A. (2002). Early Intervention: A Global Perspective. Infants and Young Children, 15(2), 11-19.

Borntrager, C. F., Chorpita, B. F., Orimoto, T., Love, A., \& Mueller, C. W. (2013). Validity of Clinician’s Self-Reported Practice Elements on the Monthly Treatment and Progress Summary. Journal of Behavioral Health Services \& Research, 42(3), 367-382. https://doi.org/10.1007/s11414-013-9363-X

Bowden, A., Fox-Rushby, J. A., Nyandieka, L., \& Wanjau, J. (2002). Methods for pre-testing and piloting survey questions: illustrations from the KENQOL survey of health-related quality of life. Health Policy and Planning, 17(3), 322-330. https://doi.org/10.1093/heapol/17.3.322

Brookman-Frazee, L., Haine, R. A., Baker-Ericzén, M., Zoffness, R., \& Garland, A. F. (2010). Factors associated with use of evidence-based practice strategies in usual care youth psychotherapy. Administration and Policy in Mental Health and Mental Health Services Research, 37(3), 254-269.

Burke, J. D., Loeber, R., \& Birmaher, B. (2002). Oppositional defiant disorder and conduct disorder: A review of the past 10 years, part II. Journal of the American Academy of Child \& Adolescent Psychiatry, 41(11), 1275-1293. https://doi.org/10.1097/01.CHI.0000024839.60748.E8 
Chirdkiatgumchai, V., Xiao, H., Fredstrom, B. K., Adams, R. E., Epstein, J. N., Shah, S. S., \& Froehlich, T. E. (2013). National trends in psychotropic medication use in young children: 1994-2009. Pediatrics, 132(4), 615-623.

Chorpita, B. F., \& Daleiden, E. L. (2009). Mapping evidence-based treatments for children and adolescents: application of the distillation and matching model to 615 treatments from 322 randomized trials. Journal of Consulting and Clinical Psychology, 77(3), 566-79. https://doi.org/10.1037/a0014565

Dillman, D. A., Smyth, J. D., \& Christian, L. M. (2014). Internet, Phone, Mail, and Mixed-Mode Surveys: The Tailored Design Method. Wiley. Retrieved from https://books.google.com/books?hl=en\&lr=\&id=W5I_BAAAQBAJ\&pgis=1

Dishion, T. J., Shaw, D., Connell, A., Gardner, F., Weaver, C., \& Wilson, M. (2008). The family check-up with high-risk indigent families: Preventing problem behavior by increasing parents’ positive behavior support in early childhood. Child Development, 79(5), 13951414. https://doi.org/10.1111/j.1467-8624.2008.01195.x

Dubow, E. F., \& Luster, T. (1990). Adjustment of children born to teenage mothers: The contribution of risk and protective factors. Journal of Marriage and the Family, (393404).

Easterbrooks, M. A., Biesecker, G., \& Lyons-Ruth, K. (2000). Infancy predictors of emotional availability in middle childhood: the roles of attachment security and maternal depressive symptomatology. Attachment \& Human Development, 2(2), 170-187. https://doi.org/10.1080/14616730050085545 
Fashimpar, G. A. (2001). Problems of Parenting: Solutions of Science. Journal of Family Social Work, 5(2), 67-80. https://doi.org/10.1300/J039v05n02_06

Fearon, R. P., Bakermans-Kranenburg, M. J., van IJzendoorn, M. H., Lapsley, A. M., \& Roisman, G. I. (2010). The significance of insecure attachment and disorganization in the development of children's externalizing behavior: A meta-analytic study. Child Development, 81(2), 435-456. https://doi.org/10.1111/j.1467-8624.2009.01405.x

Frambach, R. T., \& Schillewaert, N. (2002). Organizational innovation adoption: A multi-level framework of determinants and opportunities for future research. Journal of Business Research, 55(2), 163-176. https://doi.org/10.1016/S0148-2963(00)00152-1

Garland, A. F., Bickman, L., \& Chorpita, B. F. (2010). Change what? Identifying quality improvement targets by investigating usual mental health care. Administration and Policy in Mental Health and Mental Health Services Research, 37(1-2), 15-26. https://doi.org/10.1007/s10488-010-0279-y

Garland, A. F., Brookman-Frazee, L., Hurlburt, M. S., Accurso, E. C., Zoffness, R. J., HaineSchlagel, R., \& Ganger, W. (2010). Mental health care for children with disruptive behavior problems: a view inside therapists’ offices. Psychiatric Services (Washington, D.C.), 61(8), 788-795. https://doi.org/10.1176/appi.ps.61.8.788

Garland, A. F., Hurlburt, M. S., Brookman-Frazee, L., Taylor, R. M., \& Accurso, E. C. (2010). Methodological challenges of characterizing usual care psychotherapeutic practice. Administration and Policy in Mental Health and Mental Health Services Research, 37(3), 208-220. https://doi.org/10.1007/s10488-009-0237-8 
Garland, A. F., Landsverk, J. L., Hough, R. L., \& Ellis-MacLeod, E. (1996). Type of maltreatment as a predictor of mental health service use for children in foster care. Child Abuse \& Neglect, 20(8), 675-688. https://doi.org/http://dx.doi.org/10.1016/01452134(96)00056-7

Hawaii. (2008). CAMHD Provider Monthly Summary Instructions and Codebook. Retrieved July 1, 2013, from http://hawaii.gov/health/mental-health/camhd/library/pdf/paf/paf001.pdf

Hoagwood, K., \& Kolko, D. J. (2009). Introduction to the special section on practice contexts: A glimpse into the nether world of public mental health services for children and families. Administration and Policy in Mental Health and Mental Health Services Research, 36(1), 35-36.

Huey, S. J., \& Polo, A. J. (2008). Evidence-based psychosocial treatments for ethnic minority youth. Journal of Clinical Child and Adolescent Psychology: The Official Journal for the Society of Clinical Child and Adolescent Psychology, American Psychological Association, Division 53, 37(1), 262-301. https://doi.org/10.1080/15374410701820174

Hurlburt, M. S., Garland, A. F., Nguyen, K. \& Brookman-Frazee, L. (2010). Child and Family Therapy Process : Concordance of Therapist and Observational Perspectives, 230-244. https://doi.org/10.1007/s10488-009-0251-X

Jensen-Doss, A., \& Hawley, K. M. (2010). Understanding barriers to evidence-based assessment: clinician attitudes toward standardized assessment tools. Journal of Clinical Child and Adolescent Psychology : The Official Journal for the Society of Clinical Child and 
Adolescent Psychology, American Psychological Association, Division 53, 39(6), 88596. https://doi.org/10.1080/15374416.2010.517169

Kolko, D. J. (2006). Commentary: Studying Usual Care in Child and Adolescent Therapy: It’s Anything but Routine. Clinical Psychology: Science and Practice, 13(1), 47-52. https://doi.org/10.1111/j.1468-2850.2006.00005.x

Lim, A., Nakamura, B. J., Higa-McMillan, C. K., Shimabukuro, S., \& Slavin, L. (2012). Effects of workshop trainings on evidence-based practice knowledge and attitudes among youth community mental health providers. Behaviour Research and Therapy, 50(6), 397-406.

Llewellyn, G., McConnell, D., Honey, A., Mayes, R., \& Russo, D. (2003). Promoting health and home safety for children of parents with intellectual disability: A randomized controlled trial. Research in Developmental Disabilities, 24(6), 405-431. https://doi.org/10.1016/j.ridd.2003.06.001

Loeber, R., Green, S. M., Keenan, K., \& Lahey, B. B. (1995). Which Boys Will Fare Worse? Early Predictors of the Onset of Conduct Disorder in a Six-Year Longitudinal Study. Journal of the American Academy of Child \& Adolescent Psychiatry, 34(4), 499-509. https://doi.org/10.1097/00004583-199504000-00017

Lunkenheimer, E. S., Dishion, T. J., Shaw, D. S., Connell, A. M., Gardner, F., Wilson, M. N., \& Skuban, E. M. (2008). Collateral benefits of the Family Check-Up on early childhood school readiness: indirect effects of parents’ positive behavior support. Developmental Psychology, 44(6), 1737-52. https://doi.org/10.1037/a0013858

Macdonald, E., Mohay, H., Sorensen, D., Alcorn, N., McDermott, B., \& Lee, E. (2005). Current delivery of infant mental health services: are infant mental health needs being met? 
Australasian Psychiatry : Bulletin of Royal Australian and New Zealand College of Psychiatrists, 13(4), 393-398. https://doi.org/10.1111/j.1440-1665.2005.02232.x

Malik, N. M., Crowson, M. M., Lederman, C. S., \& Osofsky, J. D. (2002). Evaluating maltreated infants, toddlers, and preschoolers in dependency court. Infant Mental Health Journal, 23(5), 576-592. https://doi.org/10.1002/imhj.10034

McCabe, K., \& Yeh, M. (2009). Parent-child interaction therapy for Mexican Americans: a randomized clinical trial. Journal of Clinical Child and Adolescent Psychology: The Official Journal for the Society of Clinical Child and Adolescent Psychology, American Psychological Association, Division 53, 38(5), 753-759.

https://doi.org/10.1080/15374410903103544

McCabe, K., Yeh, M., Lau, A., \& Argote, C. B. (2012). Parent-Child Interaction Therapy for Mexican Americans: Results of a Pilot Randomized Clinical Trial at Follow-up. Behavior Therapy, 43(3), 606-618. https://doi.org/10.1016/j.beth.2011.11.001

McLeod, B. D., \& Weisz, J. R. (2010). The therapy process observational coding system for child psychotherapy strategies scale. Journal of Clinical Child \& Adolescent Psychology, 39(3), 436-443.

Mercer, J. (2013). Holding therapy: a harmful mental health intervention. Focus on Alternative and Complementary Therapies, 18(2), 70-76. https://doi.org/10.1111/fct.12024

Nelson, T. D., \& Steele, R. G. (2007). Predictors of practitioner self-reported use of evidencebased practices: Practitioner training, clinical setting, and attitudes toward research. Administration and Policy in Mental Health and Mental Health Services Research, 34(4), 319-330. https://doi.org/10.1007/s10488-006-0111-X 
Nicolaidis, C., Raymaker, D., McDonald, K., Dern, S., Ashkenazy, E., Boisclair, C., ... Baggs, A. (2011). Collaboration strategies in nontraditional community-based participatory research partnerships: lessons from an academic-community partnership with autistic self-advocates. Progress in Community Health Partnerships : Research, Education, and Action, 5(2), 143-150. https://doi.org/10.1353/cpr.2011.0022

NREPP, National Registry of Evidence-based Programs and Practices (2008). Active Parenting (4th Edition). Retrieved April 10, 2016, from http://legacy.nreppadmin.net/ViewIntervention.aspx?id=110

Olds, D. L. (2006). The nurse-family partnership: An evidence-based preventive intervention. Infant Mental Health Journal, 27(1), 5-25. https://doi.org/10.1002/imhj.20077

Olds, D. L., Robinson, J., O’Brien, R., Luckey, D. W., Pettitt, L. M., Henderson, C. R., ... Talmi, A. (2002). Home visiting by paraprofessionals and by nurses: a randomized, controlled trial. Pediatrics, 110(3), 486-496.

Olds, D. L., Sadler, L., \& Kitzman, H. (2007). Programs for parents of infants and toddlers: Recent evidence from randomized trials. Journal of Child Psychology and Psychiatry and Allied Disciplines, 48(3-4), 355-391. https://doi.org/10.1111/j.1469-7610.2006.01702.x

Pitzer, M., Esser, G., Schmidt, M. H., \& Laucht, M. (2010). Early predictors of antisocial developmental pathways among boys and girls. Acta Psychiatrica Scandinavica, 121(1), 52-64. https://doi.org/10.1111/j.1600-0447.2009.01411.x

Rogers, E. M. (2010). Diffusion of innovations (4th ed.). New York, NY: Simon and Schuster. 
Sameroff, A. J. (1998). Environmental risk factors in infancy. Pediatrics, 102(5 Suppl E), 12871292. https://doi.org/10.1542/peds.102.5.SE1.1287

Sanders, M. R., Prinz, R. J., \& Shapiro, C. J. (2009). Predicting utilization of evidence-based parenting interventions with organizational, service-provider and client variables. Administration and Policy in Mental Health and Mental Health Services Research, 36(2), 133-143. https://doi.org/10.1007/s10488-009-0205-3

Shaw, D., \& Dishion, T. (2006). Randomized trial of a family-centered approach to the prevention of early conduct problems: 2-year effects of the family check-up in early childhood. Journal of Consulting and Clinical Psychology, 74, 1.

Shaw, D. S. (2015). The Long-Term Effectiveness of the Family Check-Up on School-Age Conduct Problems: Moderation by Neighborhood Deprivation. Development and Psychopathology, XXXIII(2), 81-87. https://doi.org/10.1007/s13398-014-0173-7.2

Shaw, D. S., Dishion, T. J., Supplee, L., Gardner, F., \& Arnds, K. (2006). Randomized trial of a family-centered approach to the prevention of early conduct problems: 2-year effects of the family check-up in early childhood. Journal of Consulting and Clinical Psychology, 74(1), 1-9. https://doi.org/10.1037/0022-006X.74.1.1

Shaw, D. S., Gilliom, M., Ingoldsby, E. M., \& Nagin, D. S. (2003). Trajectories leading to school-age conduct problems. Developmental Psychology, 39(2), 189-200. https://doi.org/10.1037/0012-1649.39.2.189

Shaw, D. S., Owens, E. B., Giovannelli, J., \& Winslow, E. B. (2001). Infant and toddler pathways leading to early externalizing disorders. Journal of the American Academy of Child \& Adolescent Psychiatry, 40(1), 36-43. 
Sroufe, L. A., Carlson, E. A., Levy, A. K., \& Egeland, B. (1999). Implications of attachment theory for developmental psychopathology. Development and Psychopathology, 11(1), 1-13. https://doi.org/10.1017/S0954579499001923

Tymchuk, A. J., \& Andron, L. (1992). Project parenting: Child interactional training with mothers who are mentally handicapped. Mental Handicap Research, 5(1), 4-32. https://doi.org/10.1111/j.1468-3148.1992.tb00168.x

U.S. Department of Health and Human Services. (2015). Implementing Family Check-Up® For Children. Retrieved from https://homvee.acf.hhs.gov/Implementation/3/Family-CheckUp-For-Children-Implementation/9

Waters, E., Hamilton, C. E., \& Weinfield, N. S. (2000). The stability of attachment security from infancy to adolescence and early adulthood: General introduction. Child Development, 71(3), 678-683. 


\section{Table 1}

Clinician use of intervention strategies with infants and their families

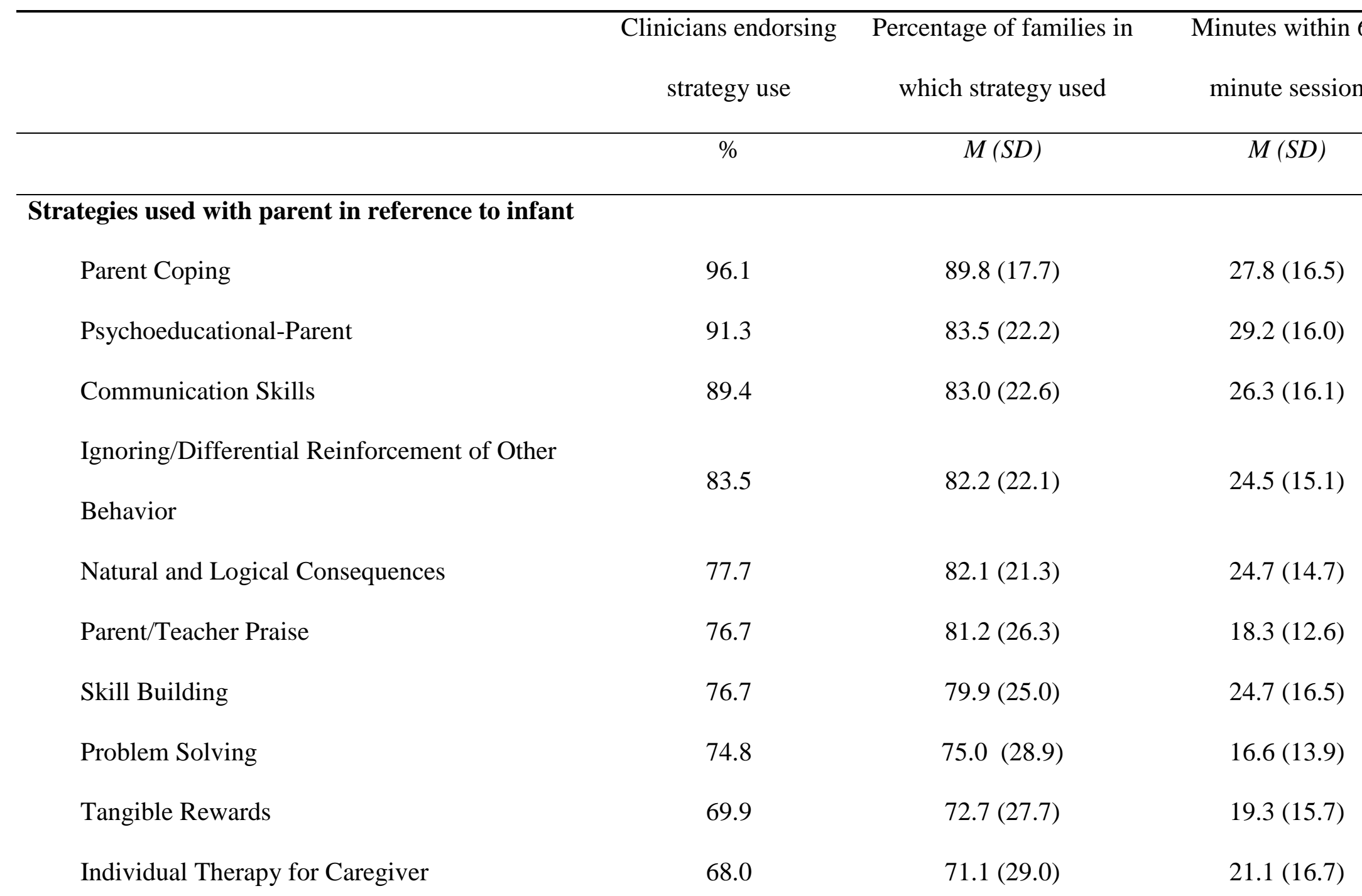




\begin{tabular}{|c|c|c|c|}
\hline Crisis Management & 66.3 & $70.5(26.4)$ & $22.1(13.8)$ \\
\hline Parent/Teacher Monitoring & 65.0 & $69.7(27.7)$ & $18.5(14.4)$ \\
\hline Activity Scheduling & 63.5 & $68.6(30.3)$ & $21.6(15.2)$ \\
\hline Goal Setting & 62.5 & $68.2(26.6)$ & $16.8(12.9)$ \\
\hline Commands & 61.0 & $67.2(27.0)$ & $19.1(14.1)$ \\
\hline Emotional Processing & 56.4 & $65.8(30.1)$ & $15.7(12.7)$ \\
\hline Stimulus/Antecedent Control & 56.3 & $64.5(27.6)$ & $17.8(16.5)$ \\
\hline Cognitive & 52.9 & $63.6(27.7)$ & $15.6(11.7)$ \\
\hline Mindfulness & 49.0 & $63.0(28.1)$ & $14.6(11.1)$ \\
\hline Motivational Interviewing & 36.3 & $62.1(30.7)$ & $19.9(13.0)$ \\
\hline Response Prevention & 31.7 & $60.2(29.0)$ & $24.6(18.4)$ \\
\hline Response Cost & 23.0 & $60.1(28.7)$ & $12.8(13.2)$ \\
\hline Catharsis & 15.7 & $49.1(32.4)$ & 17.7 (16.9) \\
\hline \multicolumn{4}{|c|}{ ategies used with parent and infant } \\
\hline Family Engagement & 87.0 & $79.3(26.5)$ & $32.4(17.2)$ \\
\hline Family Therapy & 76.6 & $70.3(31.9)$ & $33.2(18.4)$ \\
\hline
\end{tabular}


Therapist Praise/Rewards

Play Therapy

Attending

Relaxation

Maintenance

Time Out

\section{Strategies used directly with infant or general}

\section{strategy}

Relationship/Rapport Building

Care Coordination

Supportive Listening

Discrete Trial Training

Exposure

Medication/ Pharmacotherapy
75.5

74.2

64.1

64.0

44.6

39.1

95.5

79.8

78.7

16.9

14.8

6.7
68.2 (32.9)

66.7 (33.6)

67.4 (30.6)

$61.4(33.0)$

$58.9(32.2)$

$44.0(32.8)$

20.8 (16.7)

36.0 (16.5)

$27.8(17.5)$

17.3 (11.8)

20.3 (12.9)

$13.0(14.0)$

89.3 (22.9)

38.5 (17.2)

$65.4(32.1)$

$17.1(14.1)$

40.4 (16.7)

28.4 (13.6)

32.3 (26.7)

18.5 (12.1)

$43.2(25.5)$

$20.6(18.4)$ 


\section{Table 2}

Factors which typically influence clinician choice of intervention strategy

\begin{tabular}{lcc}
\hline & Frequency $^{\mathrm{a}}$ & Mean rank $\mathrm{b}^{\mathrm{b}}$ \\
\cline { 2 - 3 } & $\%(N)$ & $M(S D)$ \\
\hline Family culture & $91.3 \%(84)$ & $2.4(1.1)$ \\
Participant knowledge of intervention strategy & $88.0 \%(81)$ & $3.2(1.3)$ \\
Caregiver/child cognitive ability & $83.7 \%(77)$ & $3.4(1.3)$ \\
Results of assessment & $80.4 \%(74)$ & $2.1(1.3)$ \\
Empirical support for intervention strategy & $67.4 \%(62)$ & $3.1(1.6)$ \\
Use of intervention strategy by respected & & $5.4(1.2)$ \\
colleagues & $38.0 \%(35)$ & $5.0(2.5)$ \\
Use of intervention strategy court-ordered & $10.9 \%(10)$ & $1.8(1.0)$ \\
Other (e.g., level of family stress) ${ }^{\mathrm{c}}$ & $8.7 \%(8)$ & \\
\hline
\end{tabular}

Note. ${ }^{\mathrm{a}} n=92 .{ }^{\mathrm{b}}$ When rank ordering, a lower number is more influential. ${ }^{\mathrm{c}}$ Participants were given the option to write in an influential factor and it was included in the items to be rank ordered. 


\section{Table 3}

Percentage of providers endorsing use of program with infants and their families

\begin{tabular}{|c|c|c|c|c|}
\hline & Never & Occasionally & Very Often & Always \\
\hline Active Parenting & 39.5 & 19.8 & 29.1 & 11.6 \\
\hline $\begin{array}{l}\text { Al's Pals: Kids Making Healthy } \\
\text { Choices }\end{array}$ & 94.0 & 6.0 & 0.0 & 0.0 \\
\hline Chicago Parent Program & 100.0 & 0.0 & 0.0 & 0.0 \\
\hline Child-Parent Psychotherapy (CPP) & 44.7 & 16.5 & 28.2 & 10.6 \\
\hline Children in Between & 100.0 & 0.0 & 0.0 & 0.0 \\
\hline Circle of Security $^{\mathrm{a}}$ & 62.4 & 15.3 & 16.5 & 5.9 \\
\hline Conscious Discipline $^{\mathrm{a}}$ & 60.2 & 22.9 & 13.3 & 3.6 \\
\hline DARE to be You & 96.3 & 3.7 & 0.0 & 0.0 \\
\hline $\begin{array}{l}\text { Early HeartSmarts Program for } \\
\text { Preschool Children }\end{array}$ & 91.5 & 3.7 & 4.9 & 0.0 \\
\hline $\begin{array}{l}\text { Families and Schools Together } \\
\text { (FAST) }^{\mathrm{a}}\end{array}$ & 90.2 & 6.1 & 2.4 & 1.2 \\
\hline Family Check-Up & 95.1 & 3.7 & 1.2 & 0.0 \\
\hline Family Foundations & 96.3 & 3.7 & 0.0 & 0.0 \\
\hline Family Spirit & 100.0 & 0.0 & 0.0 & 0.0 \\
\hline FRIENDS Program & 98.8 & 1.2 & 0.0 & 0.0 \\
\hline Greenspan Floortime Approach & 64.6 & 17.1 & 17.1 & 1.2 \\
\hline Head Start ${ }^{\mathrm{a}}$ & 63.9 & 22.9 & 10.8 & 2.4 \\
\hline $\begin{array}{l}\text { Healthy Alternatives for Little Ones } \\
\text { (HALO) }\end{array}$ & 96.3 & 0.0 & 3.7 & 0.0 \\
\hline Healthy Families America ${ }^{a}$ & 89.0 & 6.1 & 3.7 & 1.2 \\
\hline Healthy Start ${ }^{\mathrm{a}}$ & 72.0 & 15.9 & 9.8 & 2.4 \\
\hline HighScope Curriculum & 90.2 & 8.5 & 1.2 & 0.0 \\
\hline HOMEBUILDERS & 96.3 & 2.4 & 0.0 & 1.2 \\
\hline I Can Problem Solve (ICPS) & 92.7 & 3.7 & 3.7 & 0.0 \\
\hline Incredible Years & 73.2 & 20.7 & 6.1 & 0.0 \\
\hline
\end{tabular}




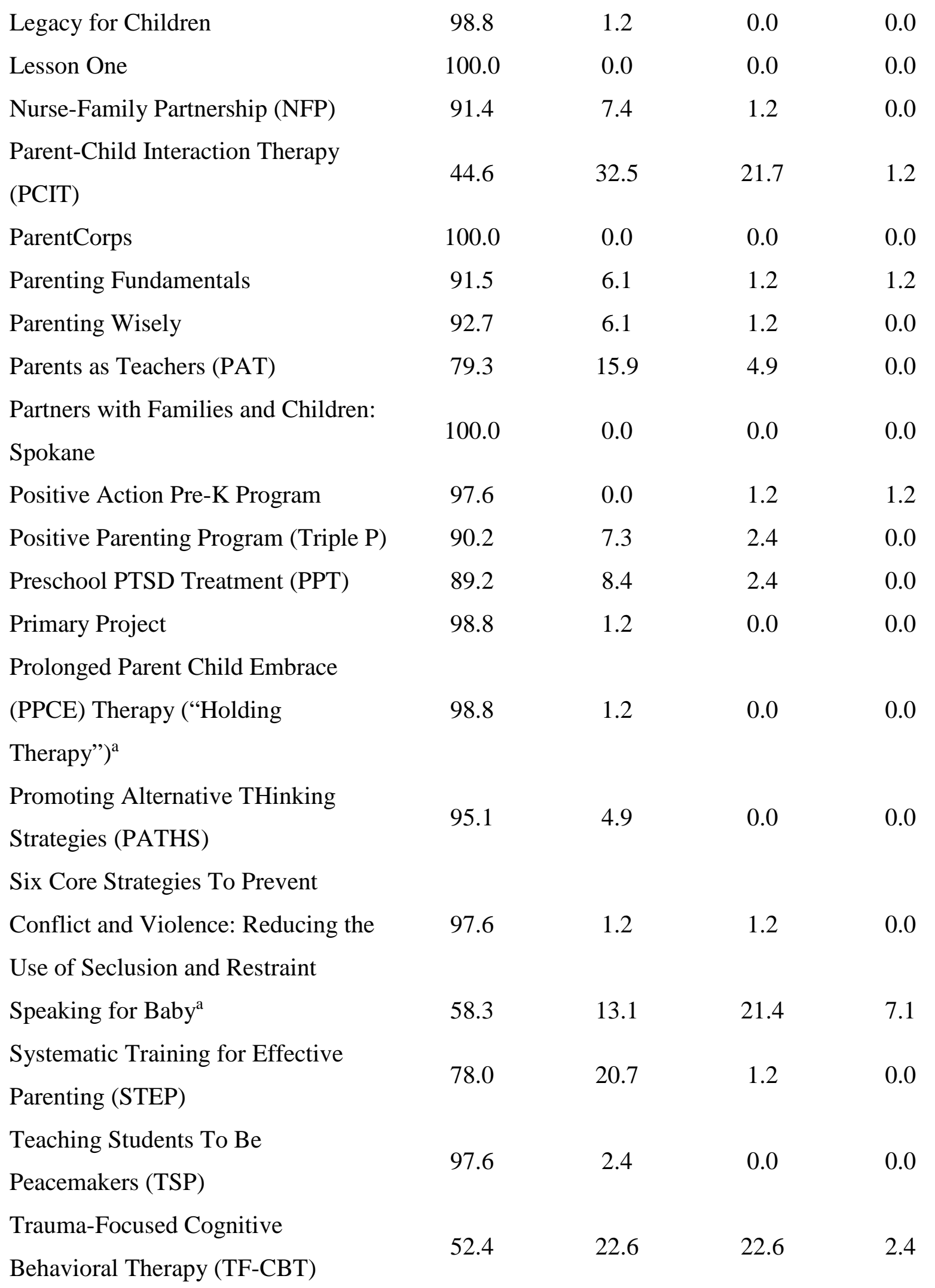


Two Families Now: Effective

$\begin{array}{lllll}\text { Parenting Through Separation and } & 100.0 & 0.0 & 0.0 & 0.0\end{array}$

Divorce (TFN)

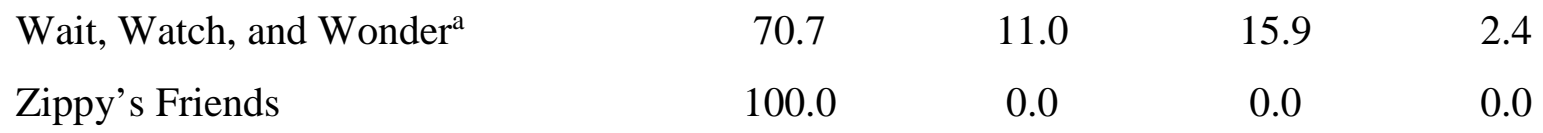

Notes. Programs obtained from the National Registry of Evidence-based Programs and Practices (NREPP) online database unless otherwise stated. ${ }^{a}$ Program suggested by discussion group participants. 


\section{Appendix A}

$\mathrm{KEY}=$ Bolded text was added during adaptation process.

Erossed out text was removed during adaptation process.

Removed strategies are crossed out entirely.

\section{$\underline{\text { Hawaii CAMHD Intervention Strategies }}$}

1. Activity Scheduling - The assignment or request that a child participate in specific activities outside of therapy time, with the goal of promoting or maintaining involvement in satisfying and enriching experiences.

z. Assertiveness Training Exercises or techniques designed to promote the child's ability to be assertive with others, ustally involving rehearsal of assertive interactions.

3. Attending - Exercises involving the youth and caregiver playing together in a specific manner to facilitate their improved verbal communication and nonverbal interaction. Can involve the caregiver's imitation and participation in the youth's activity, as well as parent-directed play (previously called "Directed Play").

4. Behavioral Contracting Development of a formal agreement specifying rules, consequences, and a commitment by the youth and relevant others to honor the content of the agreement.

5. Biofeedback/Neurofeedback Strategies to provide information about physiological activity that is typically below the threshold of perception, often involving the use of specialized equipment.

6. Care Coordination - Coordinating among the youth's service providers to ensure effective communication, receipt of appropriate services, adequate housing, etc.

7. Catharsis - Strategies designed to bring about the release of intense emotions, with the intent to develop mastery of affect and conflict.

8. Cognitive - Any techniques designed to alter interpretation of events through examination of the ehild's parent's reported thoughts, typically through the generation and rehearsal of alternative counter-statements. This can sometimes be accompanied by exercises designed to comparatively test the validity of the original thoughts and the alternative thoughts through the gathering or review of relevant information.

9. Commands - Training for caregivers in how to give directions and commands in such a manner as to increase the likelihood of child compliance.

10. Communication Skills - Training for youth or caregivers in how to communicate more effectively with others to increase consistency and minimize stress. Can include a variety of specific communication strategies (e.g., active listening, "I” statements).

11. Crisis Management - Immediate problem solving approaches to handle urgent or dangerous events. This might involve defusing an escalating pattern of behavior and emotions either in person or by telephone, and is typically accompanied by debriefing and follow-up planning. 
12. Cultural Training Education or interaction with culturally important values, rituals, or sites with no-specific practices identified.

13. Discrete Trial Training - A method of teaching involving breaking a task into many small steps and rehearsing these steps repeatedly with prompts and a high rate of reinforcement.

14. Educational Support Exercises designed to assist the child with specific academic problems, such as homework or stuly skilts. This includes tutoring.

15. Emotional Processing - A program based on an information processing model of emotion that requires activation of emotional memories in conjunction with new and incompatible information about those memories.

16. Exposure - Techniques or exercises that involve direct or imagined experience with a target stimulus, whether performed gradually or suddenly, and with or without the therapist's elaboration or intensification of the meaning of the stimulus.

17. Eye Movement/Tapping A method in which the youth is guided through a procedure to access and resolve troubling experiences and emotions, while being exposed to a therapeutic visual or tactile stimulus designed to facilitate bilateral brain activity.

18. Family Engagement - The use of skills and strategies to facilitate family or child's positive interest in participation in an intervention.

19. Family Therapy - A set of approaches designed to shift patterns of relationships and interactions within a family, typically involving interaction and exercises with the youth, the caregivers, and sometimes siblings.

20 . Free Association Technique for probing the unconscious in which a person recites a funning commentary of thoughts and feelings as they occur.

21. Functional Analysis Arrangement of antecedents and consequences based on a functional understanding of a youth's behavior. This goes beyond straightforward application of other behavioral techniques.

22. Goal Setting - Setting specific goals and developing commitment from youth or family caregivers to attempt to achieve those goals (e.g., academic, career, etc.). Revised $07 / 01 / 20087$

23. Guided Imagery - Visualization or guided imaginal techniques for the purpose of mental rehearsal of successful performance. Guided imagery for the purpose of physical relaxation (e.g., picturing calm scenery) is not coded here, but rather coded under relaxation (\#50).

24. Hypnosis The induction of a trance like mental state achieved through suggestion.

25. Ignoring/Differential Reinforcement of Other Behavior - The training of parents or others involved in the social ecology of the child to selectively ignore mild target behaviors and selectively attend to alternative behaviors.

26. Individual Therapy for Caregiver - Any therapy designed directly to target individual (non-dyadic) psychopathology in one or more of the youth's caregivers. If the therapy for caregivers involves marital therapy (\#31) or communication skills (\#10) those are not 
coded here, unless there are additional services for individual caregiver psychopathology, in which case all that apply should be coded.

27. Insight Building Activity designed to help a youth achieve greater self-understanding.

28. Interpretation-Reflective discussion or listening exercises with the child designed to yield therapeutic interpretations. This does not involve targeting specific thoughts and their alternatives, which would be coded as cognitive/coping.

29. Line of Sight Supervision-Direct observation of a youth for the purpose of assuring safe and appropriate behavior.

30. Maintenance/Relapse Prevention - Exercises and training designed to consolidate skills already developed and to anticipate future challenges, with the overall goal to minimize the chance that gains will be lost in the future

31. Marital Therapy Techniques used to improve the quality of the relationship between earegivers.

32. Medication/ Pharmacotherapy - Any use of psychotropic medication to manage emotional, behavioral, or psychiatric symptoms.

33. Mentoring Pairing with a more senior and experienced individual who serves as a positive role model for the identified youth.

34. Milieu Therapy A therapeutic approach in residential settings that involves making the environment itself part of the therapeutic program. Often involves a system of privileges and restrictions such as a token or point system.

35. Mindfulness - Exercises designed to facilitate present-focused, non-evaluative observation of experiences as they occur, with a strong emphasis of being "in the moment.” This can involve the youth's caregiver's conscious observation of feelings, thoughts, or situations.

36. Modeling Demonstration of a desired behavior by a therapist, confederates, peers, of other actors to promote the imitation and subsequent performance of that behavior by the identified youth.

37. Motivational Interviewing - Exercises designed to increase readiness to participate in additional therapeutic activity or programs. These can involve cost-benefit analysis, persuasion, or a variety of other approaches.

38. Natural and Logical Consequences - Training for parents or teachers in (a) allowing youth to experience the negative consequences of poor decisions or unwanted behaviors, or (b) delivering consequences in a manner that is appropriate for the behavior performed by the youth.

39. Parent Coping - Exercises or strategies designed to enhance caregivers' ability to deal with stressful situations, inclusive of formal interventions targeting one or more caregiver.

40. Parent/Teacher Monitoring - The repeated measurement of some target index by the parent, teacher, or other adult involved in the child's social ecology. 
41. Parent/Teacher Praise - The training of parents, teachers, or other adults involved in the social ecology of the child in the administration of social rewards to promote desired behaviors. This can involve praise, encouragement, affection, or physical proximity.

42. Peer Pairing Pairing with another youth of same or similar age to allow for reciprocal learning or skills practice.

43. Personal Safety Skills Training for the youth in how to maintain personal safety of one's physical self. This can include education about attending to one's sense of danger, body ownership issues (e.g., "good touch-bad touch"), risks involved with keeping secrets, how to ask for help when feeling unsafe, and identification of other high-risk situations for abuse.

44. Physical Exercise - The engagement of the youth in energetic physical movements to promote strength or endurance or both. Examples can include running, swimming, weight lifting, karate, soccer, etc. Note that when the focus of the physical exercise is also to produce talents or competence and not just physical activity and conditioning, the code for "Skill Building” (\#55) can also be applied.

45. Play Therapy - The use of play as a primary strategy in therapeutic activities. This may include the use of play as a strategy for clinical interpretation. Different from Attending (\#3), which involves a specific focus on modifying parent-child communication. This is also different from play designed specifically to build relationship quality (\#49).

46. Problem Solving - Techniques, discussions, or activities designed to bring about solutions to targeted problems, usually with the intention of imparting a skill for how to approach and solve future problems in a similar manner.

47. Psychoeducational-Child The formal review of information with the child about the development of a problem and its relation to a proposed intervention.

48. Psychoeducational-Parent - The formal review of information with the caregiver(s) about the development of the child's problem and its relation to a proposed intervention. This often involves an emphasis on the caregiver's role in either or both.

49. Relationship/Rapport Building - Strategies in which the immediate aim is to increase the quality of the relationship between the youth and the therapist. Can include play, talking, games, or other activities.

50. Relaxation - Techniques or exercises designed to induce physiological calming, including muscle relaxation, breathing exercises, meditation, and similar activities. Guided imagery exclusively for the purpese of physical relaxation is also coded here.

51. Response Cost - Training parents or teachers how to use a point or token system in which negative behaviors result in the loss of points or tokens for the youth.

52. Response Prevention - Explicit prevention of a maladaptive behavior that typically occurs habitually or in response to emotional or physical discomfort.

53. Self-Monitoring The repeated measurement of some target index by the child. 
54. Self-Reward/Self-Praise -Techniques designed to encourage the youth to self-administer positive consequences contingent on performance of target behaviors. Revised $07 / 01 / 20089$

55. Skill Building - The practice or assignment to practice or participate in activities with the intention of building and promoting talents and competencies.

56. Social Skills Training Providing information and feedback to improve interpersonal verbal and non-verbal functioning, which may include direct rehearsal of the skills. If this is paired with peer pairing (\#42), that should be coded as well.

57. Stimulus/Antecedent Control - Strategies to identify specific triggers for problem behaviors and to alter or eliminate those triggers in order to reduce or eliminate the behavior.

58. Supportive Listening - Reflective discussion with the child designed to demonstrate warmth, empathy, and positive regard, without suggesting solutions or alternative interpretations.

59. Tangible Rewards - The training of parents or others involved in the social ecology of the child in the administration of tangible rewards to promote desired behaviors. This can involve tokens, charts, or record keeping, in addition to first-order reinforcers.

60. Therapist Praise/Rewards - The administration of tangible (i.e., rewards) or social (e.g., praise) reinforcers by the therapist.

61. Thought Field Therapy Techniques involving the tapping of various parts of the body in particular sequences or "algorithms" in order to correct unbalanced energies, known as thought fields.

62. Time Out - The training of or the direct use of a technique involving removing the youth from all reinforcement for a specified period of time following the performance of an identified, unwanted behavior.

63. Twelve-Step Program - Any programs that involve the twelve-step model for gaining control over problem behavior, most typically in the context of alcohol and substance use, but can be used to target other behaviors as well 